INPLASY

PROTOCOL

To cite: He et al. The effect of auricular point pressing therapy on postoperative pain of total hip arthroplasty: A protocol for systematic review and meta-analysis. Inplasy protocol 202210015. doi: 10.37766/inplasy2022.1.0015

Received: 04 January 2022

Published: 04 January 2022

Corresponding author: Jie He

sg_jiejie243@126.com

Author Affiliation:

Jiangyan hospital affiliated to Nanjing University of Chinese Medicine.

Support: None.

Review Stage at time of this submission: Preliminary searches.

Conflicts of interest: None declared.

\section{The effect of auricular point pressing therapy on postoperative pain of total hip arthroplasty: A protocol for systematic review and meta-analysis}

He, J1; Ma, L2; Zhou, F3; Jiang, $\mathrm{H}^{4}$.

Review question / Objective: This systematic review aims to evaluate the effect of auricular point pressing therapy on postoperative pain of total hip arthroplasty.

Condition being studied: Total hip arthroplasty (THA) is a common surgical treatment for end-stage hip osteoarthritis and femoral neck fractures. In US, more than one million patients undergo THA treatment every year. Limited evidence suggests that auricular point pressing therapy relieve pain with fewer adverse effects.

Information sources: Relevant studied will be searched in the following electronic databases: PubMed, EMBASE, Cochrane Library, CINAHL, China Knowledge Resource Integrated Database, Weipu Database for Chinese Technical Periodicals, Sinomed, and Wanfang Database. The search terms include auricular, point, points, total hip replacement, total hip arthroplasty.

INPLASY registration number: This protocol was registered with the International Platform of Registered Systematic Review and Meta-Analysis Protocols (INPLASY) on 04 January 2022 and was last updated on 04 January 2022 (registration number INPLASY202210015).

\section{INTRODUCTION}

Review question / Objective: This systematic review aims to evaluate the effect of auricular point pressing therapy on postoperative pain of total hip arthroplasty.
Condition being studied: Total hip arthroplasty (THA) is a common surgical treatment for end-stage hip osteoarthritis and femoral neck fractures. In US, more than one million patients undergo THA treatment every year. Limited evidence suggests that auricular point pressing 
therapy relieve pain with fewer adverse effects.

\section{METHODS}

Participant or population: Participants with total hip arthroplasty accept the treatment of auricular point pressing therapy. There are no limits to research subjects' age, gender, condition duration.

Intervention: The experimental group was treated with auricular point pressing therapy.

Comparator: The control group was treated with placebo, drugs, or other alternative therapy.

Study designs to be included: All randomized controlled trials investigating the effect of auricular point pressing therapy on postoperative pain of total hip arthroplasty in Chinese and English will be enrolled without publication status restriction.

Eligibility criteria: Designs such as animal experiments, reviews, quasi-RCTs, case reports, and laboratory studies will be rejected. The ongoing studies or unpublished data will not be included.

Information sources: Relevant studied will be searched in the following electronic databases: PubMed, EMBASE, Cochrane Library, CINAHL, China Knowledge Resource Integrated Database, Weipu Database for Chinese Technical Periodicals, Sinomed, and Wanfang Database. The search terms include auricular, point, points, total hip replacement, total hip arthroplasty.

Main outcome(s): The primary outcome of interest includes visual analogue scale (VAS), numeric rating scale (NRS). The secondary outcomes include adverse reactions, patient satisfaction.

Quality assessment / Risk of bias analysis: Two reviewers will evaluate the methodological quality with the Cochrane Collaboration Tool independently. The quality evaluation items include these items: random sequence generation, allocation concealment, the blinding method for patients, researchers and outcomes assessors, incomplete outcome data, and selective reports. According to the criteria, the study will be classed as "low risk of bias", "high risk of bias", "unclear risk of bias".

Strategy of data synthesis: The research will be performed by Review Manager version 5.3 software. $P<0.05$ will be defined as statistically significant between studies. Heterogeneity will be evaluated by $I^{2}$ statistic. When $I^{22}$ is $<50 \%$, it is acceptable and a fixed effect model will be used for data analysis. When $\mathrm{I}^{2} 2$ is $>50 \%$, we will try to find the reason for the high heterogeneity through subgroup or sensitivity analysis. If there is no obvious clinical or methodological heterogeneity, the random effects model will be applied for analysis.

Subgroup analysis: If the meta-analysis shows significant heterogeneity in the studies, we will perform a subgroup analysis based on type of intervention, controls, and outcome measurements to find out the source of heterogeneity.

Sensitivity analysis: The sensitivity analysis will be conducted to test the stability of results of meta-analysis by the study of large weight of elimination effect.

Language: Without any language or publication status restrictions.

Country(ies) involved: China.

Keywords: total hip arthroplasty, auricular point pressing therapy, protocol, systematic review.

Contributions of each author:

Author 1 - Jie He - The author drafted the manuscript.

Author 2 - Lin Ma - The author provided statistical expertise.

Author 3 - Feng Zhou - The author contributed to the development of the 
selection criteria, and the risk of bias assessment strategy.

Author 4 - Hongbo Jiang - The author contributed to the development of the selection criteria, and the risk of bias assessment strategy. 